Revue d'histoire de l'Amérique française

BSW REVUE D.HISTOIRE DE L'AMÉRIQUE FRANÇAISE

\title{
La réglementation du contenu des actes de baptême, mariage, sépulture, au Québec, des origines à nos jours
}

\section{Gérard Bouchard et André LaRose}

Volume 30, numéro 1, juin 1976

URI : https://id.erudit.org/iderudit/303510ar

DOI : https://doi.org/10.7202/303510ar

Aller au sommaire du numéro

Éditeur(s)

Institut d'histoire de l'Amérique française

ISSN

0035-2357 (imprimé)

1492-1383 (numérique)

Découvrir la revue

Citer cette note

Bouchard, G. \& LaRose, A. (1976). La réglementation du contenu des actes de baptême, mariage, sépulture, au Québec, des origines à nos jours. Revue

d'histoire de l'Amérique française, 30(1), 67-84. https://doi.org/10.7202/303510ar d'utilisation que vous pouvez consulter en ligne. 


\section{LA RÉGLEMENTATION DU CONTENU DES ACTES DE BAPTÊME, MARIAGE, SÉPULTURE, AU QUÉBEC, DES ORIGINES À NOS JOURS}

GÉRARD BOUCHARD

Département des sciences bumaines

Université du Québec à Chicoutimi

ANDRÉ LAROSE

Département de démographie

Université de Montréal

Il n'est guère de démographes ou d'historiens qui ne connaissent aujourd'hui les efforts pionniers de Louis Henry et de Pierre Goubert qui ont fait des registres de baptêmes, mariages et sépultures un instrument privilégié pour la connaissance des populations anciennes ${ }^{1}$. La réputation de cette source n'est pas surfaite. Pour être lente et lourde, son exploitation n'en ouvre pas moins la voie à des analyses très précises de matières aussi diverses que la santé, la vie familiale, les rapports sociaux, les mœurs, sans oublier bien sûr les rubriques propres à l'analyse démographique proprement dite. On constate cependant que, sous l'influence des réglementations, le contenu de cette documentation a varié selon les lieux et les époques. Ce facteur peut restreindre sensiblement l'éventail des analyses possibles et il appelle une étude soigneuse des dispositions juridiques qui ont régi la tenue des registres.

De la Nouvelle-France d'hier au Québec d'aujourd'hui, comment le contenu des actes a-t-il évolué ? C'est à cette question que

\footnotetext{
L. Henry, «Une richesse démographique en friche: les registres paroissiaux», Population, 8e année, no 2 (avril-juin 1953): 281-290; P. Goubert, "Une richesse historique en cours d'exploitation: les registres paroissiaux », Annales. Économies, Sociétés, Civilisations, vol. 9, no 1 (janvier-mars 1954): 83-93.
} 
nous tenterons de répondre ici ${ }^{2}$ en reconstituant l'évolution des réglementations religieuse et civile en matière d'enregistrement des baptêmes, mariages et sépultures. Étant donné l'ampleur du sujet et les nécessités du moment, nous avons dû limiter cet examen aux prescriptions fixant le contenu des trois types d'actes. Pour chacun d'eux, nous nous sommes livrés à une rétrospective des principaux textes de loi, en dressant à chaque fois, sous forme de tableau, la liste des renseignements demandés par le législateur. Il est facile de voir ensuite en quoi chaque intervention modifie les dispositions antérieures et dans quel sens elle altère le contenu des actes.

Nous espérons par là faire progresser la connaissance de cette source fondamentale et, peut-être, faciliter la tâche à d'autres chercheurs. Nous fixons ainsi la norme à laquelle sont soumis, à chaque époque, les rédacteurs des actes. Ce n'est évidemment là qu'une démarche préliminaire; reste ensuite à déterminer, dans le cadre d'enquêtes locales ou régionales, dans quelle mesure les curés et autres officiers de l'état civil se sont conformés aux règles prescrites.

Pour le bénéfice des lecteurs peu familiers avec ces questions, il y a lieu de rappeler que les registres paroissiaux, comme leur nom l'indique, sont d'origine ecclésiastique. Ils apparurent en France et en Italie au XIV ${ }^{\mathrm{e}}$ siècle mais ne se généralisèrent qu'au XVII ${ }^{\mathrm{e}}$. En 1563, le Concile de Trente étendit à l'Église universelle l'obligation d'enregistrer les baptêmes et les mariages qui n'était jusque-là le fait que de quelques diocèses. Mais ce n'est qu'avec la parution du Rituale romanum en 1614 que s'ajouta l'enregistrement des sépultures, celui des confirmations et celui de l'état des âmes ${ }^{3}$.

2 Les auteurs de cet article font partie de deux équipes de chercheurs engagés dans des projets d'histoire démographique. Gérard Bouchard est, conjointement avec Yolande Lavoie, responsable du Projet d'histoire sociale de la population du Saguenay, à l'Université du Québec à Chicoutimi. André LaRose collabore depuis quelques années au Programme de recherche en démographie historique (P.R.D.H.) du Département de démographie de l'Université de Montréal, que dirigent Hubert Charbonneau et Jacques Légaré. Les auteurs remercient de leur appui financier leurs bailleurs de fonds respectifs, soit le Conseil des Arts du Canada, pour le projet de Chicoutimi, le programme Killam du Conseil des Arts et le programme F.C.A.C. du Ministère de l'Éducation du Québec, pour le P.R.D.H.

3 L'état des âmes (status animarum) est la liste nominative que dressent les curés au cours de leur visite paroissiale. - Sur l'histoire des registres paroissiaux en Europe, voir Roger Mols, s.j., Introduction à la démographie historique des villes d'Europe du XIV e au XVIII ${ }^{e}$ siècle (Gembloux, J. Duculot S.A., et Louvain, Bibliothèque de l'Université, 1954-56), I: 75-102; Jacques Levron, "Les registres paroissiaux et d'état civil en France», Archivum. vol. IX (1959): 55-100; René Le Mée, «La réglementation des registres paroissiaux en France», Société de démographie 
Si les registres paroissiaux ont à l'origine servi aux curés à tenir leurs comptes des honoraires perçus lors des cérémonies, c'est cependant une raison d'ordre canonique qui permet d'en expliquer la diffusion. Ces registres servaient en effet à déceler les empêchements aux mariages dus à la parenté naturelle ou à l'affinité spirituelle ${ }^{3 a}$. L'enregistrement des mariages a en outre mis un frein à un abus: celui des mariages clandestins ${ }^{4}$.

Le pouvoir civil ne manqua pas lui non plus de s'intéresser à l'enregistrement des baptêmes, mariages et sépultures et son intervention en cette matière fit des registres un champ de juridiction mixte. La préoccupation de l'État d' «établir clairement les points essentiels se rapportant à la condition juridique des personnes ${ }^{5}$ se manifesta en France dans les ordonnances de Villers-Cotterêts (1539), Blois (1579), Saint-Germain-en-Laye (1667) et dans la Déclaration royale de 1736. Les ordonnances de 1539 et 1579 ne créèrent pas l'enregistrement; elles eurent plutôt pour effet de reconnaître officiellement les registres catholiques. Parce qu'ils constituaient la preuve la plus recevable des naissances, des mariages et des décès, les registres paroissiaux français firent donc pleine foi de l'état des personnes au for civil de 1579 à 1792, date de l'institution d'un état civil distinct ${ }^{6}$.

Au Canada, les registres paroissiaux firent leur apparition dès l'arrivée des premiers missionnaires qui répondaient ainsi aux exigences du Concile de Trente et à celles du Rituel aussi bien qu'à celles des ordonnances de 1539 et 15797 . À la requête du Conseil souverain, les registres furent tenus en double à partir de $1679^{8}$ et l'avènement du régime anglais ne modifia pas cette habitude. Bien

historique. Annales de démographie historique 1975: 433-472; André LaRose, Les registres paroissiaux au Québec aux XVII" et XVIII" siècles: introduction à l'étude d'une institution religieuse et civile. Mémoire de M.A. (histoire), (Université de Montréal, mars 1976), 6-20.

3a L'affinité spirituelle est la parenté par alliance contractée dans le baptême entre le parrain et la marraine, entre ceux-ci et leur filleul(e) de même qu'entre ceux-ci et les parents de leur filleul(e).

4 Mols, op. cit., I: 86; LaRose, op. cit., 8-9.

5 Mols, op. cit., I: 163.

6 Roméo Lemelin, ptre, «Les registres de l'état civil des personnes dans la province de Québec", Revue de l'Université Laval, I, no 9 (mai 1947): 712-713. $21-31$

7 Sur l'histoire des registres paroissiaux au Québec, voir LaRose, op. cit.:

8 L'ordonnance de 1667 n'a été enregistrée à Québec que le 7 novembre 1678 et elle entra en vigueur en janvier suivant. Ibid., 26. 
plus, l'Assemblée législative du Bas-Canada sanctionna cet usage et l'étendit même aux congrégations protestantes, dans une loi de 1795 qui s'inspirait directement de l'ordonnance de 1667 et de la Déclaration royale de 1736. Le Code civil du Bas-Canada (1866) reprit essentiellement les anciennes lois, de telle sorte qu'aujourd'hui encore au Québec, les registres de baptêmes, mariages, sépultures tenus par les curés sont en même temps des registres d'état civil. Les registres paroissiaux continuent donc de faire l'objet d'une double juridiction et c'est pourquoi notre comparaison des réglementations ecclésiastique et civile va des origines à nos jours. Signalons cependant que, compte tenu de l'évolution sociale actuelle, l'Assemblée nationale du Québec instaurera sous peu un état civil distinct, suivant les recommandations de l'Office de révision du Code civil ${ }^{9}$.

Il nous a paru commode de livrer nos résultats en deux parties, la première consacrée à la réglementation ecclésiastique; la seconde à la réglementation civile. Dans l'une et l'autre, nous passons en revue les trois types d'actes et reconstituons l'évolution de leur conteniu. Nos commentaires s'appuient sur les tableaux que l'on trouvera en appendice.

\section{I - Le droit ecclésiastique}

Il n'est guère à propos de faire ici une récapitulation minutieuse des ordonnances des autorités diocésaines concernant les actes de baptême, mariage et sépulture ${ }^{10}$. Les fréquentes interventions des évêques, des synodes et des conciles recouvrent une tradition juridique relativement stable, comme nous le verrons. Plusieurs textes ne concernent pas le contenu des actes comme tel, d'autres viennent notifier aux curés et vicaires tel amendement de la loi civile ou leur signaler certaines négligences et omissions. Nous nous en tiendrons donc à quelques textes essentiels.

C'est d'abord le Concile de Trente qui, en 1563, imposa à l'ensemble de l'Église l'enregistrement des baptêmes et des mariages. Il sanctionnait du même coup une pratique déjà ancienne mais dont seuls quelques diocèses s'étaient occupés jusque-là "'. Soucieux avant

9 Québec (Province). Office de révision du Code civil. Rapport sur l'ćtat civil/Report on Civil Status (Montréal, 1973), 124 p. "Rapports des Comités soumis à l'Office de révision du Code civil, no 23 ».

10 Pour une liste abrégée, voir François Beaudin, «Les registres paroissiaux au Québec», Archives 69.2 (juillet-décembre 1969): 29.

11 Le Mée, loc. cit., 442; Cyprien Tanguay, Registres de l'état des personnes (Ottawa, 1878), $19 \mathrm{p}$. 
tout de vérifier la légitimité des naissances et de combattre les mariages clandestins, le Concile avait omis de prescrire la rédaction d'actes de sépulture. Cette lacune fut comblée en 1614 par le Rituale romanum, qui apportait en même temps de substantielles additions aux énoncés précédents. Par la suite, les synodes diocésains ont intégré ces normes aux usages locaux ${ }^{12}$. Le rituel, guide de l'administration des sacrements et des diverses cérémonies du culte, donna alors lieu à de nombreuses versions, subissant au chapitre des formules, maintes adaptations mineures.

Les premiers registres paroissiaux du Québec remontent précisément à cette période. Il est difficile de déterminer avec précision les règles auxquelles cet enregistrement s'est conformé à l'origine, l'Église de Nouvelle-France n'ayant pas publié de directives formelles sur ce sujet avant le début du XVIII ${ }^{\mathrm{e}}$ siècle. Il est cependant probable que les premiers ecclésiastiques français établis dans la colonie ont continué d'appliquer les règles de leur diocèse d'origine c'est-à-dire, pour l'essentiel du moins, les prescriptions du Rituale romanum. Ce n'est qu'en 1703 que fut publié, sur ordre de Mgr de Saint-Vallier, le premier rituel canadien ${ }^{13}$. Celui-ci prescrivait des formules pour la rédaction des actes de baptême, mariage et sépulture dont la précision des énoncés est exemplaire, compte tenu de l'époque. Au chapitre des sépultures toutefois, il accusait des déficiences qui ne furent corrigées qu'en $1836^{14}$. Avant cette date, en effet, on ne relève aucun amendement aux vieux formulaires, ce qui pour une part atteste leur qualité.

Les éditions successives de l'Appendice au Rituel romain de 1853 et de 1874 reprennent en tous points le texte de 1836 . Seules les éditions de 1890 et 1919 y introduisent quelques modifications superficielles. Cette dernière fait d'ailleurs suite à la publication en 1918 du Codex Iuris Canonici où est déterminé, pour l'Église universelle, l'essentiel de ce qui doit être consigné dans les actes ${ }^{15}$. Le Code rappelle en outre aux curés ${ }^{16}$ l'obligation qu'ils ont, depuis le décret

12 Mols, op. cit., I: 89.

13 Rituel du diocèse de Québec (Paris, 1703). Pour plus de détails sur ce qui précède, voir LaRose, op. cit., chap. II.

14 Extrait du Rituel de Québec (Québec, 1836), 308-314.

15 Codes Iuris Canonici... (New York. P.J. Kenedy \& Sons, MCMXVIII), canons 470.2, 777.1, 1103 et 1238 .

16 Ibid., canon 470.2 . 
Ne temere du 2 août $1907^{17}$, de noter en marge de l'acte de baptême qu'un individu a été confirmé, a contracté mariage, a reçu le sousdiaconat ou a fait profession solennelle ${ }^{18}$. Ces annotations ne peuvent évidemment être faites que sur les registres demeurant dans les archives paroissiales. Soulignons cependant que le Rituel, auquel renvoie le Code, est plus complet que celui-ci puisqu'il contient des formules adaptées aux exigences locales.

Il convient donc de souligner la remarquable prévoyance dont a fait preuve la tradition ecclésiastique, ce qui se reflète du reste dans la constance des prescriptions et énoncés. Dès lors, on ne saisit pas bien les motifs qui ont animé les auteurs de la réforme récente. Depuis 1973 en effet, l'Assemblée des évêques du Québec, par souci d'uniformité sans doute, a ordonné l'usage de nouveaux formulaires pour les trois types d'actes. On peut voir, par les tableaux I et V surtout, que les nouvelles dispositions ont amené un appauvrissement de la matière à enregistrer. Sur un plan strictement scientifique, la réforme de 1973 opère donc un recul qu'il convient de déplorer ${ }^{19}$.

\section{II - Le droit civil}

Il faut remonter aux vieilles ordonnances royales des $X \mathrm{I}^{\mathrm{e}}$ et $\mathrm{XVII}{ }^{\mathrm{e}}$ siècles pour trouver l'origine de la juridiction civile en vigueur au début de la colonie. C'est d'abord l'ordonnance de Villers-Cotterêts qui institua au civil l'enregistrement de l'état des personnes. Le roi étendait de cette façon à l'ensemble du Royaume certaines pratiques en usage dans quelques diocèses. Mais les préoccupations de l'État faisaient la part belle aux problèmes de succession, de contrats et de transmission des biens matériels. Il fut ainsi enjoint aux curés et vicaires de France de dresser des actes, pour toutes les naissances survenant en leur paroisse, de manière à établir sûrement la capacité

17 W. van Ommeren, «Ne temere», New Catholic Encyclopaedia (New York, McGraw Hill, 1967), X: 288; Roméo Lemelin, Les registres paroissiaux dans la province civile de Qućbec (Dissertation soumise à la Faculté de Droit canonique de l'Université Laval, Québec, 1944), 69.

18 P. Torquebiau, "Baptême en Occident», dans R. Naz, Dictionnaire de droit canonique (Paris, Letouzey et Ané, 1937), II : col. 172; Lemelin, op. cit. : 295-309.

19 Cf. Mgr Marius Paré, Lettre circulaire à propos des actes des registres (Chicoutimi, 21 novembre 1972), Annexes II et III. Au total, trois éléments nouveaux sont intégrés aux formulaires pendant que dix sont retranchés. Il y a même lieu de se demander si le formulaire des baptêmes est tout à fait conforme aux dispositions des articles 54 et 56 du Code civil actuel, relatives au sexe du nouveau-né et aux enfants illégitimes ou de parents inconnus. 
des personnes, et pour les sépultures d'ecclésiastiques pourvus de bénéfices ${ }^{20}$. Dans les deux cas, le célébrant devait faire état avec précision de la date de la naissance ou du décès ainsi que des noms et prénoms des parties ${ }^{21}$.

L'ordonnance de Blois en 1579 reprit ces dispositions à son compte mais en bloc et d'une manière indirecte seulement. Mise à part la présence de quatre témoins au mariage, ce texte ne contient pas de prescriptions explicites quant au contenu des actes ${ }^{22}$. Il faut toutefois porter à son crédit le fait d'avoir prescrit l'enregistrement du mariage; l'article 181 laisse néanmoins supposer que les registres de mariages et de sépultures existaient déjà ${ }^{23}$. C'est en 1667 , avec l'ordonnance de Saint-Germain-en-Laye, que des modifications majeures survinrent quant au contenu. Tous les actes allaient désormais porter la signature des parties, du moins sur le registre demeurant en paroisse. À cela s'ajoutaient notamment d'importantes mentions d'âge et de domicile ${ }^{24}$.

En les reprenant textuellement le 7 novembre 1678, le Conseil souverain de Québec mettait ces prescriptions en vigueur dans la colonie canadienne. Elles le demeurèrent jusqu'en 1727, date à laquelle le Conseil supérieur de la Nouvelle-France opérait à son tour une importante réforme. Le nouveau règlement précisait la formule de l'acte de mariage (ex.: la signature du célébrant) et surtout ordonnait de substantielles additions aux actes de sépulture, comme l'âge et la profession du défunt ${ }^{25}$. L'ordonnance de Louis XV en 1736 ne reprit pas toutes ces dispositions, s'attachant principalement à augmenter le contenu des actes de mariage, auquel s'intégraient d'importantes mentions, telles la profession et le domicile des témoins ${ }^{26}$. On a l'assurance que cette dernière réforme a été reconnue

20 Sur ce sujet, se rapporter à P.-B. Mignault, Le droit civil canadien (Montréal, 1895), I: 164.

21 Le Mée, loc. cit.: 440 et 443.

22 Le père Mols semble s'être mépris sur ce point en rapportant de prétendues stipulations de l'ordonnance. Voir R. Mols, «Les registres paroissiaux sous l'Ancien Régime», Nouvelle revue théologique, 78, no 5 (mai 1956): 510.

23 Le Mée, loc. cit. : 443.

24 Lemelin, "Les registres de l'état civil des personnes dans la province de Québec», Revue de l'Univ'ersité Lav'al, I, no 10 (juin 1947): 822-823.

25 Bulletin des recherches historiques, XXXIX, no 7 (juillet 1933): 415-426. cité par LaRose, op. cit., 163-165,

26 Lemelin, loc. cit. : 830. 
en Nouvelle-France ${ }^{27}$; et comme elle n'a vraisemblablement pas annulé les ajouts de 1727 , il est donc permis de supposer que, jusqu'à la fin du XVIII ${ }^{\text {e }}$ siècle, les registres de la colonie l'ont emporté en rigueur et en précision sur ceux de la métropole.

La Déclaration de 1736 «a cessé d'être en vigueur dans le civil pendant bien des années entre la conquête et l'époque du Bill de Québec », écrit Mgr D'Esglis en $1785^{28}$. À la requête du lieutenantgouverneur, l'avocat du roi a cependant statué cette année-là qu'elle subsistait «dans toute sa force» depuis la promulgation de l'Acte de Québec en $1774^{29}$. On constate cependant que ce n'est qu'à partir de 1786 que le pouvoir civil a effectivement remis en application l'ancienne loi française ${ }^{30}$.

La réglementation traditionnelle fut enfin reformulée en 1795 dans une loi de l'Assemblée législative du Bas-Canada ${ }^{31}$. Cette loi, qui a la réputation d'avoir prolongé les vieilles ordonnances royales, modifiait sensiblement le contenu des trois actes, mais sans opérer de progrès spectaculaire. Par rapport au Règlement de 1727, elle ajoutait six mentions et en retranchait trois ; par rapport à l'ordonnance de Louis $\mathrm{XV}$, elle en ajoutait six également mais en enlevait autant. L'avance semble donc incertaine, à plus forte raison si on compare ces énoncés à ceux du Code Napoléon promulgué en France à la même époque ${ }^{32}$. Ce rapprochement laisse voir l'écart qui avait commencé à se creuser entre la tradition juridique canadienne sous

27 LaRose, op. cit., 27-28. Voir aussi J.-Jacques Loranger. Commentaire sur le code civil du Bas-Canada (Montréal, 1873-1879), I: 417.

2r H. Têtu et C.O. Gagnon, Mandements, lettres pastorales et circulaires des évêques de Québec (Québec, Côté, 1888), II : 319.

29 Ibid.

30 LaRose, op. cit., 27-30.

31 Il s'agit du "Registration Act», par lequel la législation civile en matière d'enregistrement des baptêmes, mariages et sépultures était étendue aux Églises et aux congrégations protestantes. En même temps, les registres sommaires qu'elles avaient tenus dans le passé étaient reconnus légalement. Cf. Ibid., 30. Cependant, les ministres protestants ont mis du temps à se conformer aux nouvelles directives, ce qui explique la qualité très inégale des registres non catholiques jusqu'à la fin du XIX ${ }^{\mathrm{e}}$ siècle au moins. Là-dessus, voir Robert R. Kuczynski, Birth Registration and Birth Statistics in Canada (Washington, 1930), chapitres I et II ; D. Girouard, "Nos registres de l'état civil», Bulletin des recherches historiques, XXXII, no 4 (avril 1926): 220221 ; «Registres protestants et registres catholiques », Ibid. : 231-232.

32 Voir en particulier les énoncés très riches des articles 76 et 79 du Code Napoléon. 
le régime anglais et le droit français ${ }^{33}$. On ne peut donc pas dire que la législation canadienne se soit appauvrie après la Conquête. puisque au contraire il s'y est marqué une certaine évolution. Mais celle-ci eût été beaucoup plus nette, incontestablement, sans la rupture avec la tradition française.

Les codificateurs de 1866 , il est vrai, ont remédié en partie au laconisme de leurs prédécesseurs en précisant l'énoncé des actes de baptême et de mariage (cf. articles 54-56, 64 et 65 du Code civil). Au total cependant, le code québécois est demeuré très inférieur au Code Napoléon sous ce rapport.

Nous avons déjà suggéré qu'en pratique, selon les époques et les lieux, les officiers ont infléchi en des sens très divers les dispositions de la loi. Par exemple, il est assuré que les curés n'ont pas attendu 1795 pour inscrire la date des mariages. Pourtant, avant cette année, on ne trouve pas d'énoncé prescrivant formellement cette précision. De même, plusieurs mentions importantes n'ont jamais été incorporées à aucun texte de loi, y compris le Code civil actuel. Par exemple, aux actes de sépulture, le curé n'a jamais été tenu de noter le lieu du décès, de la sépulture ou de la naissance du décédé, son domicile et celui de l'autre conjoint; ou, si le décédé est un enfant, les noms et prénoms de ses parents, leur domicile, leur profession. Quant au sexe du défunt, seul l'énoncé de 1727 en fait mention d'une manière explicite.

Dans cette perspective, on comprendra aisément l'intérêt que suscitent les travaux de l'actuel Office de révision du Code civil de la province de Québec, chargé entre autres de reformuler les règles de l'enregistrement. Par l'entremise d'un comité ad hoc, l'Office a rendu public en 1973 un rapport préliminaire sur l'état civil dont certaines dispositions ne laissent pas de surprendre. Ainsi par rapport aux énoncés du Code civil en vigueur depuis 1866, les futures déclarations de naissance, mariage et décès ajoutent 7 mentions et en retranchent 14. Parmi les mentions rayées, on trouve la profession du père et de la mère d'un nouveau-né, les noms et prénoms du père et de la mère d'un enfant décédé, le nom du conjoint défunt au mariage d'un veuf, les noms et prénoms des parents des époux, la profession de toute personne décédée ${ }^{34}$.

33 André LaRose (op. cit., 31) a montré que cet écart résultait d'une attitude délibérément hostile affichée par les gouvernants du Bas-Canada à l'endroit de la France révolutionnaire et impériale.

34 Cf. Rapport sur l'état civil, articles 4, 18, 22, 28. 
Si ces recommandations avaient été adoptées, les registres de l'état civil seraient devenus presque inutilisables pour des études de mobilité socio-professionnelle; en outre, ils se seraient difficilement prêtés à la reconstitution des familles puisque la déclaration de mariage ne rattache pas les conjoints à leurs parents. Heureusement, diverses représentations ont été faites auprès du comité de l'état civil qui a consenti certaines modifications. Par exemple, les porte-parole des Archives Nationales ont obtenu que les articles 22 et 28 du texte projeté identifient clairement la famille des époux et des enfants décédés. D'autres organismes se sont fait entendre récemment, parmi lesquels le Département de démographie de l'Université de Montréal, qui a réclamé que les noms et prénoms du conjoint soient toujours connus, même si celui-ci est décédé ${ }^{35}$, et l'Institut d'histoire de l'Amérique française, qui a demandé que les trois déclarations soient plus explicites quant à la profession des personnes ${ }^{36}$. Il faut souhaiter que l'Office se montre accueillant à ce genre de requêtes qui, à tout prendre, ne visent guère plus qu'au statu quo. En de meilleurs jours eñ effet, on auiraiat pui espérer des énoncés cncore plus riches, sans qu'il en coûte beaucoup ni aux législateurs ni aux futurs officiers de l'état civil.

\section{Conclusion}

Même s'ils ne s'élèvent guère au-dessus de la simple comptabilité, les résultats de cette brève recherche permettent néanmoins de constater que du XVI ${ }^{\mathrm{e}}$ siècle à nos jours, une unité d'ensemble et une même ligne de pensée se dégagent des réglementations successives des autorités ecclésiastiques et civiles en matière d'enregistrement des actes de baptême, de mariage et de sépulture. Au Québec, le contenu actuel des actes est le fruit d'une longue tradition qui s'est maintenue sans solution de continuité depuis l'Ancien Régime. Les quelques modifications apportées avec le temps à ce contenu n'altèrent pas la valeur juridique des actes. Mais la suppression de certains éléments (illégitimité, parenté des parrain et marraine avec le baptisé, profession et domicile des parents des époux ainsi que du père ou de l'époux du défunt) peut parfois gêner les praticiens de l'histoire sociale et de la démographie historique, toujours avides de renseignements. Nous nous inquiétons d'ailleurs des tendances actuelles de la législation visant à réduire l'information à inscrire dans les actes, au détriment des chercheurs de demain.

35 Lettre de H. Charbonneau, le 28 juillet 1975.

36 Lettre du président J.-P. Wallot, le 31 octobre 1975. 
Les textes que nous avons examinés montrent que les intérêts de l'Église et de l'État pour la constatation de l'état des personnes se rejoignent. Mais la réglementation ecclésiastique a toujours été plus détaillée que la réglementation civile. Cela vient d'abord du fait qu'en plus des informations réclamées conjointement par les deux pouvoirs, le droit de l'Église demande des renseignements que ne requiert pas le droit civil parce qu'ils relèvent de la juridiction exclusive de l'Église et qu'ils n'affectent pas la condition des personnes dans la société civile. D'autre part, nos tableaux tiennent compte à la fois du droit de l'Église universelle et du droit particulier aux diocèses du Québec. Or ce dernier se manifeste dans des formules prévues pour chaque cas, alors que les ordonnances et les lois de l'autorité civile s'en tiennent à des prescriptions plus générales. Il est assez normal d'ailleurs que la réglementation ecclésiastique soit plus précise que la réglementation civile puisque ce sont les curés qui sont chargés de l'enregistrement pour les fins des deux pouvoirs. Au total, on peut dire que l'Église du Québec a eu la souplesse nécessaire pour s'adapter à cette situation et l'État en a bénéficié.

On sait maintenant précisément à quoi s'attendre lorsqu'on dépouille les registres paroissiaux du Québec. Reste à voir si les curés ont suivi les règlements à la lettre. Pour le Canada du XVII siècle et le Saguenay du XIX ${ }^{\mathrm{e}}$, l'enquête est en cours ${ }^{37}$. Ses résultats indiqueront la valeur des registres et permettront de déterminer les analyses possibles à partir de cette masse de documents. Dans la même veine, d'autres questions méritent aussi d'être soulevées: jusqu'à quel point, par exemple, les autorités ecclésiastiques et civiles ont-elles exercé un contrôle de la bonne tenue des registres ou, de façon plus large, dans quel contexte et pour quelles raisons les autorités sont-elles intervenues pour amender la réglementation des registres paroissiaux? - Ces documents, on le voit, n'ont pas fini de faire parler d'eux.

37 Voir Gérard Bouchard et Michel Bergeron, «Les registres de l'état civil de Notre-Dame de Laterrière (1855-1911) », Archives 75.3, VII, no 3 (septembre-décembre 1975): 164-173; Raymond Roy et Hubert Charbonneau, "Le contenu des registres paroissiaux canadiens du XVII ${ }^{\text {e }}$ siècle», RHAF, 30, 1 (juin 1976) : 85-97. 
TABLEAU I

Évolution du contenu des actes de baptême d'après la réglementation ecclésiastique en vigueur au Québec

- : renseignement demandé de façon explicite $\bigcirc$ : renseignement demandé de façon implicite

\begin{tabular}{|c|c|c|c|c|c|c|c|}
\hline \multirow{2}{*}{ ÉLÉMENTS DU CONTENU } & \multicolumn{7}{|c|}{ DATE DE LA RÉGLEMENTATION } \\
\hline & $\begin{array}{c}1563 \\
\text { (1) }\end{array}$ & $\begin{array}{c}1614 \\
(2)\end{array}$ & $\begin{array}{c}1703 \\
(3)\end{array}$ & $\begin{array}{c}1836 \\
(4)\end{array}$ & $\begin{array}{c}1890 \\
(5)\end{array}$ & $\begin{array}{c}1919 \\
(6)\end{array}$ & $\begin{array}{c}1973 \\
\text { (7) }\end{array}$ \\
\hline $\begin{array}{l}\text { Date de naissance } \\
\text { Date de baptême } \\
\text { Lieu de naissance } \\
\text { Lieu de baptême } \\
\text { Ondoiement }\left\{\begin{array}{l}\text { pourquoi } \\
\text { par qui }\end{array}\right.\end{array}$ & O & 0 & • & ○ & $\stackrel{\bullet}{\bullet}$ & 0 & ? \\
\hline $\begin{array}{l}\text { Nom de l'enfant } \\
\text { Sexe } \\
\text { Légitimité ou illégimité }\end{array}$ & $\stackrel{\bullet}{0}$ & ? & ? & $\begin{array}{l}\bullet \\
\bigcirc \\
\bullet\end{array}$ & ? & 0 & $\stackrel{\bullet}{0}$ \\
\hline $\begin{array}{l}\text { Noms des parents } \\
\text { Profession du père } \\
\text { Domicile des parents } \\
\text { Absence du père au baptême } \\
\text { Parents inconnus }\end{array}$ & & ○ & $\begin{array}{l}\bullet \\
0 \\
\bullet\end{array}$ & $\begin{array}{l}\bullet \\
\bullet \\
\bullet \\
\bullet \\
\bullet\end{array}$ & ? & ? & ? \\
\hline $\begin{array}{l}\text { Noms des parrain et marraine } \\
\text { Père du parrain, si celui-ci est mineur } \\
\text { Père ou mari de la marraine b } \\
\text { Procurateurs des parrain et marraine } \\
\text { Profession du parraina } \\
\text { Domicile des parrain et marraine } \\
\text { Parenté avec le baptisé }\end{array}$ & $\bullet$ & 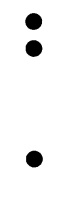 & ? & $\bullet$ & $\begin{array}{l}\bullet \\
0 \\
0 \\
0 \\
0\end{array}$ & $\stackrel{\bullet}{\bullet}$ & ? \\
\hline $\begin{array}{l}\text { Signature }\left\{\begin{array}{l}\text { du pèrec } \\
\text { des parrain et marraine } \\
\text { du célébrant }\end{array}\right. \\
\text { Ne sait pas signer }\end{array}$ & & & $\begin{array}{l}\bullet \\
\bullet \\
\bullet\end{array}$ & ? & 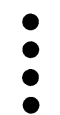 & ? & ? \\
\hline
\end{tabular}

Notes

a De nos jours, lorsque la mère et la marraine ont une profession, on l'inscrit, en principe. En 1890, on disait: "Comme la plupart des marraines n'ont pas de profession, il faut présumer que l'intention de la loi est que la marraine puisse être facilement distinguée de toute autre personne portant le même nom: on y satisfait en disant par exemple, épouse ou veuve de $N \ldots$ ou bien: grand-mère, tante, seur, cousine de l'enfant ou encore: fille de N...». Appendice au Rituel romain (Québec, 1890): 167, en note.

b Lorsque les parrain et marraine sont absents, ils sont représentés au baptême par des fondés de procuration spéciale.

c La mère signe aussi, lorsqu'elle assiste au baptême, ce qui est de plus en plus fréquent de nos jours.

SOURCES: Voir p. 83. 
TABLEAU II

Évolution du contenu des actes de baptême d'après la réglementation civile en vigueur au Québec

: renseignement demandé de façon explicite $\bigcirc$ : renseignement demandé de façon implicite

\begin{tabular}{|c|c|c|c|c|c|c|c|}
\hline \multirow{2}{*}{ ÉLÉMENTS DU CONTENU } & \multicolumn{7}{|c|}{ DATE DE LA RÉGLEMENTATION } \\
\hline & $\begin{array}{c}1539 \\
\text { (1) }\end{array}$ & $\begin{array}{c}1579 \\
(2)\end{array}$ & $\begin{array}{c}1667 \\
(3)\end{array}$ & $\begin{array}{l}1727 \\
(4)\end{array}$ & $\begin{array}{c}1736 \\
(5)\end{array}$ & $\begin{array}{c}1795 \\
(6)\end{array}$ & $\begin{array}{c}1866 \\
(7)\end{array}$ \\
\hline $\begin{array}{l}\text { Date de naissance } \\
\text { Date de baptême } \\
\text { Lieu de naissance } \\
\text { Lieu de baptême } \\
\text { Ondoiement }\left\{\begin{array}{l}\text { pourquoi } \\
\text { par qui }\end{array}\right.\end{array}$ & $\bullet$ & & • & $\bullet$ & • & $\stackrel{\bullet}{\bullet}$ & • \\
\hline $\begin{array}{l}\text { Nom de l'enfant } \\
\text { Sexe } \\
\text { Légitimité ou illégitimité }\end{array}$ & $\begin{array}{l}0 \\
0\end{array}$ & & $\stackrel{\bullet}{0}$ & $\stackrel{\bullet}{0}$ & $\stackrel{\bullet}{0}$ & ○ & $\stackrel{\bullet}{\bullet}$ \\
\hline $\begin{array}{l}\text { Noms des parents } \\
\text { Profession du pèreb } \\
\text { Domicile des parents } \\
\text { Absence du père au baptême } \\
\text { Parents inconnus }\end{array}$ & $\bigcirc$ & & ○ & - & ○ & ? & ? \\
\hline $\begin{array}{l}\text { Noms des parrain et marraine } \\
\text { Père du parrain, si celui-ci est mineur } \\
\text { Père ou mari de la marraine } \\
\text { Procurateurs des parrain et marraine } \\
\text { Profession du parrain } \\
\text { Domicile des parrain et marraine } \\
\text { Parenté avec le baptisé }\end{array}$ & & & - & - & - & - & $\begin{array}{l}0 \\
\bullet \\
\bullet\end{array}$ \\
\hline $\begin{array}{l}\text { Signature }\left\{\begin{array}{l}\text { du pèrec } \\
\text { des parrain et marraine } \\
\text { du célébrant }\end{array}\right. \\
\text { Ne sait pas signer }\end{array}$ & & & ? & ? & ? & ? & $\stackrel{?}{\bullet}$ \\
\hline
\end{tabular}

\section{Notes}

a L'obligation d'inscrire le lieu de naissance n'est apparue qu'en 1941, par suite de la multiplication des naissances ayant lieu à l'hôpital, c'est-à-dire le plus souvent en dehors des limites de la paroisse du baptisé. Statuts du Québec (1940), 4 Geo. VI, ch. 68 , art. 1.

b Depuis 1866, lorsque la mère et la marraine ont une profession, on l'inscrit, en principe. Voir Tableau I, note a.

c En 1795 et en 1866 , on demande que l'acte de baptême soit signé par le père et la mère, «s'ils sont présents». Statuts provinciaux du Bas-Canada (1795), 35 Geo. III, ch. IV, art. III; Code civil du Bas-Canada (1866), art. 55.

SOURCES: Voir p. 84. 


\section{TABLEAU III}

Evolution du contenu des actes de mariage d'après la réglementation ecclésiastique en vigueur au Québec

- renseignement demandé de façon explicite $\bigcirc$ : renseignement demandé de façon implicite

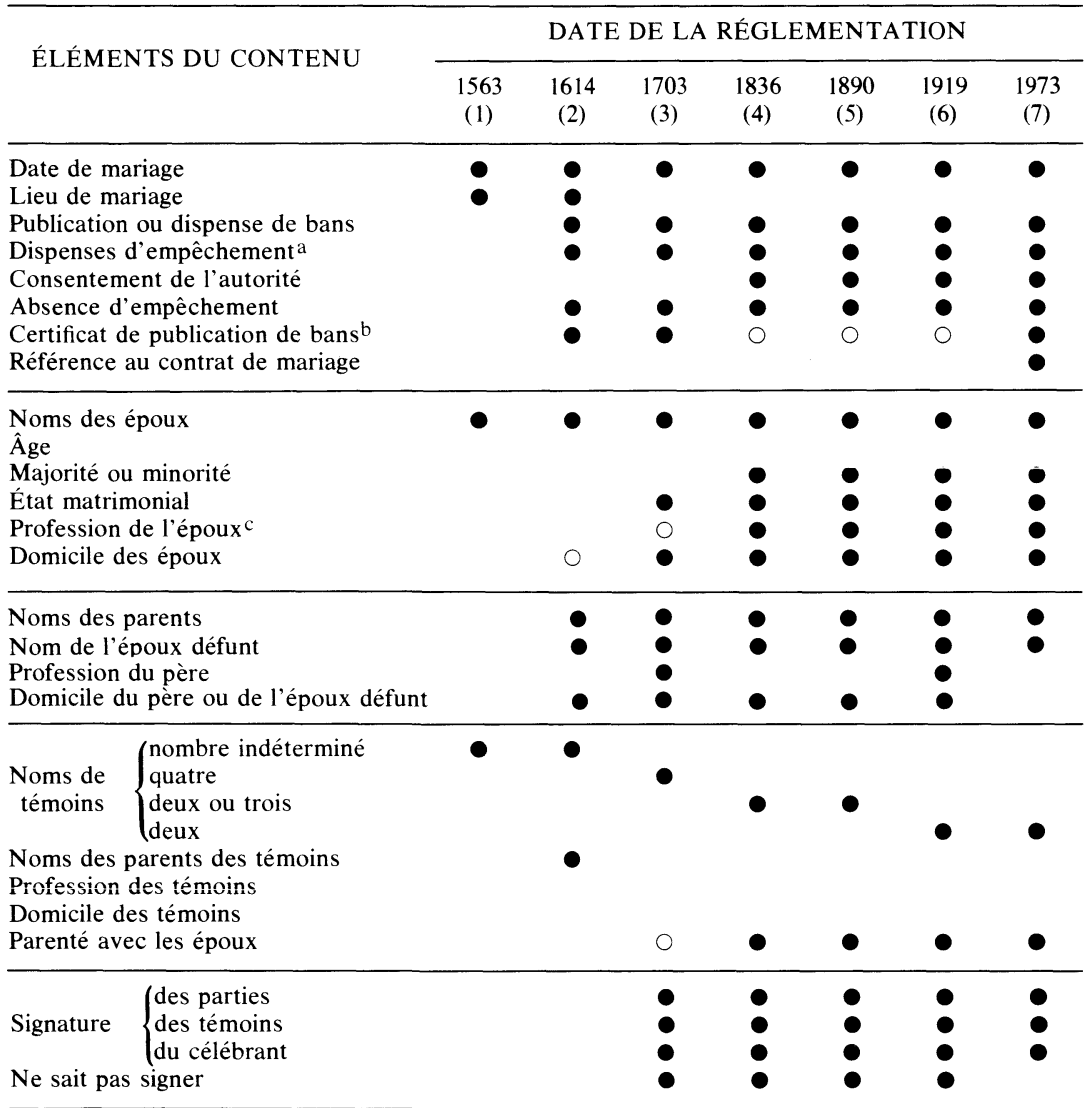

\section{NOTES}

a Aujourd'hui, les dispenses d'empêchement les plus courantes sont les dispenses de consanguinité et celles de religion mixte; autrefois, on rencontrait aussi des dispenses de temps prohibé (Avent, Carême) et d'affinité spirituelle.

b La publication de bans fait l'objet d'un certificat dont les rituels demandent habituellement la mention à l'acte, surtout si l'un des époux est d'une autre paroisse.

- De nos jours, on note aussi la profession de l'épouse, en principe.

SOURCES: Voir p. 83. 
TABLEAU IV

Évolution du contenu des actes de mariage d'après la réglementation civile en vigueur au Québec

-: renseignement demandé de façon explicite $\bigcirc$ : renseignement demandé de façon implicite

\section{ÉLÉMENTS DU CONTENU}

DATE DE LA RÉGLEMENTATION

\begin{tabular}{ccccccc}
\hline 1539 & 1579 & 1667 & 1727 & 1736 & 1795 & 1866 \\
$(1)$ & $(2)$ & $(3)$ & $(4)$ & $(5)$ & $(6)$ & $(7)$
\end{tabular}

Date de mariage

Lieu de mariage

Publication ou dispense de bans

Dispenses d'empêchement

Consentement de l'autoritéa

Absence d'empêchement ${ }^{b}$

Certificat de publication de bans

Référence au contrat de mariage ${ }^{c}$

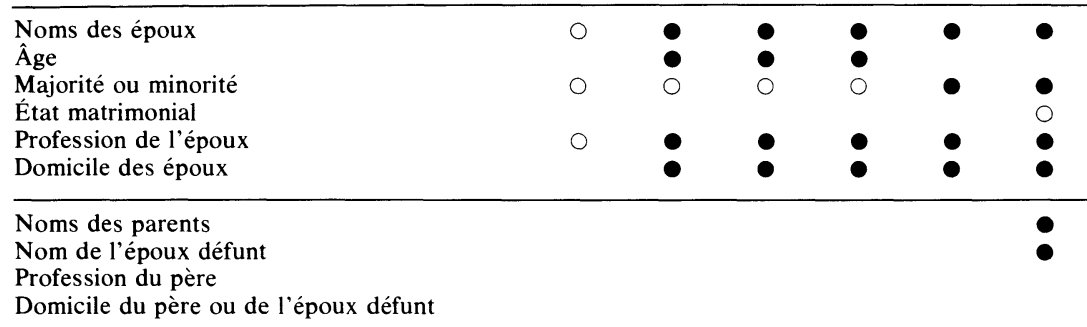

\begin{tabular}{l}
$\begin{array}{c}\text { Noms de } \\
\text { témoins }\end{array} \quad \begin{array}{l}\text { nombre indéterminé } \\
\text { quatre } \\
\text { deux ou trois } \\
\text { deux }\end{array}$ \\
$\begin{array}{l}\text { Noms des parents des témoins } \\
\text { Profession des témoins } \\
\text { Domicile des témoins } \\
\text { Parenté avec les époux }\end{array}$ \\
\hline Signature $\left\{\begin{array}{l}\text { des parties } \\
\text { des témoins } \\
\text { du célébrant }\end{array}\right.$ \\
Ne sait pas signer
\end{tabular}

\section{Notes}

a Pour se marier, les mineurs doivent avoir le consentement de leurs père et mère, tuteur ou curateur; et sous le régime français, les militaires devaient obtenir la permission du gouverneur pour le faire.

b S'il y a opposition au mariage, mainlevée doit être obtenue avant de procéder à la cérémonie. Code civil du Bas-Canada (1866), art. 61.

c Depuis 1931, il faut mentionner dans l'acte si oui ou non il y a eu contrat de mariage. $\mathrm{Si}$ oui, on indique alors le nom et l'adresse du notaire. Statuts du Québec (1930), 21 Geo. V, ch. 101, art. 1.

SOURCES: Voir p. 84. 


\section{TABLEAU V}

Évolution du contenu des actes de sépulture d'après la réglementation ecclésiastique en vigueur au Québec
-: renseignement demandé de façon explicite $\bigcirc$ : renseignement demandé de façon implicite

\begin{tabular}{lccccccc}
\hline \multirow{2}{*}{ ÉLÉMENTS DU CONTENU } & \multicolumn{7}{c}{ DATE DE LA RÉGLEMENTATION } \\
\cline { 2 - 7 } & $\begin{array}{c}1563 \\
(1)\end{array}$ & $\begin{array}{c}1614 \\
(2)\end{array}$ & $\begin{array}{c}1703 \\
(3)\end{array}$ & $\begin{array}{c}1836 \\
(4)\end{array}$ & $\begin{array}{c}1890 \\
(5)\end{array}$ & $\begin{array}{c}1919 \\
(6)\end{array}$ & $\begin{array}{c}1973 \\
(7)\end{array}$ \\
\hline Date de décès & & $\bullet$ & $\bullet$ & $\bullet$ & $\bullet$ & $\bullet$ & $\bullet$ \\
Date de sépulture & & $\bullet$ & $\bullet$ & $\bullet$ & $\bullet$ & $\bullet$ & $\bullet$ \\
Lieu de décès & $\bullet$ & $\bullet$ & $\bullet$ & $\bullet$ & $\bullet$ & $\bullet$ \\
Lieu de sépulture & $\bullet$ & $\bullet$ & $\bullet$ & $\bullet$ & $\bullet$ & $\bullet$ \\
\hline
\end{tabular}

\section{Nom du défunt \\ Sexe \\ Âge \\ État matrimonial \\ Profession \\ Domicile $^{\mathrm{a}}$ \\ Illégitimité $b$}
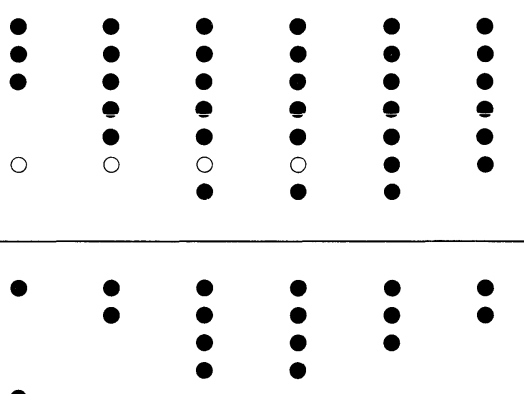

Noms des parents

Nom de l'époux (se)

Profession du père

Profession de l'époux

Domicile

Noms des témoins ${ }^{c}$

Parenté avec le défunt

Signature $\left\{\begin{array}{l}\text { des témoins } \\ \text { du célébrant }\end{array}\right.$

Ne sait pas signer

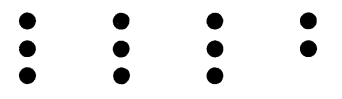

Notes

a Dans le cas des étrangers, le Rituel de Mgr de St-Vallier demande même qu'on inscrive leur lieu de naissance dans l'acte de sépulture. Rituel du diocèse de Québec, 648.

b L'illégitimité, dans les actes de sépulture, n'est relevée que dans le cas des enfants.

- La réglementation ecclésiastique demande au moins deux témoins.

SOURCES: Voir p. 83. 
TABLEAU VI

Évolution du contenu des actes de sépulture d'après la réglementation civile en vigueur au Québec

-: renseignement demandé de façon explicite $\bigcirc$ : renseignement demandé de façon implicite

\begin{tabular}{|c|c|c|c|c|c|c|c|}
\hline \multirow{2}{*}{ ÉLÉMENTS DU CONTENU } & \multicolumn{7}{|c|}{ DATE DE LA RÉGLEMENTATION } \\
\hline & $\begin{array}{c}1539^{\mathrm{a}} \\
\text { (1) }\end{array}$ & $\begin{array}{c}1579 \\
\text { (2) }\end{array}$ & $\begin{array}{c}1667 \\
(3)\end{array}$ & $\begin{array}{l}1727 \\
\text { (4) }\end{array}$ & $\begin{array}{c}1736 \\
(5)\end{array}$ & $\begin{array}{c}1795 \\
\text { (6) }\end{array}$ & $\begin{array}{c}1866 \\
(7)\end{array}$ \\
\hline $\begin{array}{l}\text { Date de décès } \\
\text { Date de sépulture } \\
\text { Lieu de décès } \\
\text { Lieu de sépulture }\end{array}$ & $\bullet$ & & $\bullet$ & 0 & $\bullet$ & $\bullet$ & • \\
\hline $\begin{array}{l}\text { Nom du défunt } \\
\text { Sexe } \\
\text { Âge } \\
\text { État matrimonial } \\
\text { Profession } \\
\text { Domicile } \\
\text { Illégitimité }\end{array}$ & 0 & & $\begin{array}{l}0 \\
0\end{array}$ & $\begin{array}{l}0 \\
\bullet \\
\bullet \\
\bullet\end{array}$ & • & ○ & $\stackrel{\bullet}{0}$ \\
\hline
\end{tabular}

Nom des parents

Nom de l'époux (se)

Profession du père

Profession de l'époux

Domicile

Noms des témoins $b$

Parenté avec le défunt

\begin{tabular}{llllll}
\hline Signature $\begin{array}{l}\text { des témoins } \\
\text { du célébrant }\end{array}$ & $\bullet$ & $\bullet$ & $\bullet$ & $\bullet$ & $\bullet$ \\
Ne sait pas signer & $\bullet$ & $\bullet$ & $\bullet$ & $\bullet$
\end{tabular}

\section{Notes}

a L'ordonnance de Villers-Cotterêts ne réclame d'acte de sépulture que pour les personnes tenant bénéfice, c'est-à-dire les évêques, les prieurs, les abbés...

b La réglementation civile demande deux témoins.

SOURCES: Voir p. 84.

Sources des tableaux I, III ET V:

(1) Concile de Trente. Canons sur la réforme touchant au mariage (Decret Tametsi), chapitre I, cité par René Le Mée, «La réglementation des registres paroissiaux en France», Annales de démographie historique 1975: 442. 
(2) Rituale Romanum (Lutetiae Parisiorum, 1623), 471-478, cité ibid.: 445-446.

(3) Rituel du diocèse de Québec (Paris, Simon Langlois, 1703), 641-648; extrait publié par André LaRose, Les registres paroissiaux au Québec aux XVII et XVIII " siècles: introduction à l'étude d'une institution religieuse et civile. Mémoire de M.A. (histoire) (Université de Montréal, mars 1976), 182-187.

(4) Extrait du Rituel de Québec (Québec, T. Cary et Cie, 1836), 308-314.

(5) Appendice au Rituel romain à l'usage des provinces écclésiastiques de Québec Montréal, Ottawa (Québec, Narcisse - S. Hardy, 1890) 166-180.

(6) Appendice au Rituel romain (Québec, Imprimerie de l'Action Sociale, Limitée, 1919), 199-212.

(7) Mgr Marius Paré, Lettre circulaire à propos des actes des registres (Chicoutimi, 21 novembre 1972), $8 \mathrm{p}$.

Sources des tableaux II, IV et VI:

(1) François 1er, Ordonnance de Villers-Cotterêts (août 1539), dans François-André Isambert, éd., Recueil général des anciennes lois françaises, depuis l'an 420 jusqu'à la Révolution de 1789 (Paris, 1822-1833); (Ridgewood, N.J., Gregg, 19641966), II, tome XII, no 188, art. 50-55: 610-611.

(2) Henri III, Ordonnance de Blois (mai 1579), ibid., II, tome XIV, 2e partie, no 103, art. 40: 391-392 et art. 181: 423.

(3) Louis XIV, Ordonnance de St-Germain-en-Iaye (avril 1667), ihid., III. tome XVIII, no 503, Titre XX: 137-140. Cette ordonnance, enregistrée au Conseil souverain de Québec, le 7 novembre 1678, a également été publié dans Edits, ordonnances royaux, déclarations et arrêts du Conseil d'Etat du roi concernant le Canada (Québec, Fréchette, 1854), 158-163, dans le BRH, XXXIX, no 5 (mai 1933): 304-307 et dans André LaRose, Les registres paroissiaux au Québec (...), 154-156.

(4) «Règlement du Conseil Supérieur de la Nouvelle-France au sujet des registres tenus par les curés (...)» (mai 1727), dans le BRH, XXXIX, no 7 (juillet 1933): 415-426 et dans A. LaRose, op. cit., 160-167.

(5) Louis XV, Déclaration concernant la forme de tenir les registres (...) (9 avril 1736), dans F. A. Isambert, op. cit., III, tome XXI, no 487: $405-416$ et dans A. LaRose, op. cit., 168-175.

(6) «Acte qui établit la forme des Régistres (sic) (...)», dans les Statuts provinciaux du Bas-Canada (1795), 35 Geo. III, ch. IV; publié dans A. LaRose, op. cit., 176-181.

(7) E. Lef. de Bellefeuille, éd., Code civil du Bas-Canada (Montréal, C. O. Beauchemin et Valois, 1866), art. 39 à 69. 